\title{
Fluência Verbal e Variáveis Sociodemográficas no Processo de Envelhecimento: Um Estudo Epidemiológico
}

\author{
Verbal Fluency and Sociodemographic Variables in the Aging Process: \\ An Epidemiological Study
}

\author{
Thaís Bento Lima da Silva, Mônica Sanches Yassuda*, Vanessa Valente Guimarães \\ \& Alex Antônio Florindo \\ Universidade de São Paulo, São Paulo, Brasil
}

\begin{abstract}
Resumo
A fluência verbal é um marcador das funções executivas, envolvendo a capacidade de busca e recuperação de dados, habilidades de organização, autorregulação e memória operacional. Objetivou-se identificar a existência de diferenças em fluência verbal (número de animais, categorias, grupos e alternância de categorias) entre sexo, faixas etárias, faixas de escolaridade e renda. Trezentos e oitenta e três idosos (60 anos ou mais) participaram de estudo epidemiológico de corte transversal. Foram aplicadas questões sociodemográficas e o teste de fluência verbal categoria animais. As variáveis do teste de fluência verbal foram influenciadas por sexo, idade e escolaridade, com melhor desempenho a favor dos homens, dos participantes mais jovens e mais escolarizados. Os resultados confirmam que o desempenho em fluência verbal deve ser interpretado à luz das informações sociodemográficas.

Palavras-chave: Fluência Verbal; Funções Executivas; Idosos; Envelhecimento.
\end{abstract}

\begin{abstract}
Verbal fluency is a marker of executive functions which involves the ability of searching and retrieving information, organizational skills, self-regulation and working memory. The objective of this paper was to identify differences in verbal fluency (number of animals, categories, clusters and category switching) associated with gender, age, education and income. Three hundred eighty three elderly (60 or older) participated in an epidemiological cross-sectional study. Participants answered sociodemographic questions and completed the verbal fluency animal category test. Verbal fluency variables were influenced by gender, age, and education. Higher performance was reported for men and participants with lower age and higher education. Results confirm that performance in verbal fluency must be interpreted in the light of sociodemographic information.

Keywords: Verbal Fluency; Executive Functions; Elderly; Aging.
\end{abstract}

O processo de envelhecimento cognitivo é caracterizado por estabilidade e mudança. Entre as habilidades que sofrem alterações normativas, associadas às mudanças fisiológicas que ocorrem no cérebro, encontram-se as funções executivas e alguns subsistemas da memória (Yassuda \& Abreu, 2006).

O termo funções executivas indica uma série de habilidades cognitivas e princípios de organização necessários para o enfrentamento das situações ambíguas do relacionamento social, e para uma conduta apropriada, responsável e efetiva (Lezak, Howieson, \& Loring 2004). As funções executivas (FE) referem-se a um conjunto de habilidades complexas que incluem a capacidade de pla-

\footnotetext{
" Endereço para correspondência: Universidade de São Paulo, Av. Arlindo Bettio, 1000, Ermelino Matarazzo, São Paulo, SP, Brasil, CEP 03828-000. Emails: yassuda@usp.br, thaisbento@usp.br, valente.guimaraes@gmail.com, e aflorind@usp.br
}

nejar, monitorar, ordenar, assim como inibir ações e processamentos indesejados (Neri, 2006; West, 1996). As funções executivas também estão associadas ao raciocínio abstrato, às características de personalidade, e à teoria da mente (Kristensen, 2006). São de extrema importância para o controle das habilidades mentais, funcionando como um maestro que ordena quando outras funções iniciarão ou cessarão suas atividades.

A fluência verbal de um indivíduo, tema de interesse do presente estudo, pode ser considerada um indicador das funções executivas, visto que envolve a capacidade de busca e recuperação de dados armazenados na memória de longo prazo. A tarefa de fluência verbal (FV) exige habilidades de organização, auto-regulação e memória operacional (Magila \& Caramelli, 2001).

No teste de fluência verbal, o entrevistado deve nomear exemplares de uma determinada categoria semântica, como animais e frutas, por um minuto, divididos em intervalos de 15 segundos. Como em outras tarefas 
cognitivas, vários aspectos da cognição são avaliados nesta tarefa de modo integrado, neste caso, atenção, riqueza de vocabulário, capacidade de inibir o processamento de outras categorias, e a realização de uma busca mental com restrições (Magila \& Caramelli, 2001).

O teste de fluência verbal, apesar de ter aplicação simples, é uma tarefa cognitiva complexa. É muito sensível a todo tipo de dano cerebral, apontando, precocemente, os processos de deterioração. O teste foi recomendado pelo Departamento Científico de Neurologia Cognitiva e do Envelhecimento, da Academia Brasileira de Neurologia (Nitrini et al., 2005) para o rastreio das demências. Aliado a testes como o Mini-Exame do Estado Mental (Brucki, Nitrini, Caramelli, Bertolucci, \& Okamoto, 2003) tem sido aplicado para determinar quais idosos apresentariam risco elevado para quadros demenciais e necessitariam de exames mais aprofundados. Alguns estudos realizados no Brasil e no exterior corroboram a utilidade do teste de fluência verbal para esta finalidade (Brucki \& Rocha, 2004; Carnero, Lendinez, Maestre, \& Zunzunegui, 1999; Gaillard et al., 2003).

Rodrigues, Yamashita e Chiappetta (2008) documentam que um pior desempenho nas tarefas de fluência verbal pode ser o resultado de alterações em pelo menos três diferentes funções cognitivas subordinadas, como memória operacional, linguagem e memória semântica. A fluência verbal é muitas vezes usada como um teste de memória verbal (armazenamento léxico e semântico), pois é necessário um processo de armazenamento e acesso das informações semânticas. A organização da informação semântica envolve os lobos temporais (E ou D), pois estudos comprovam que pacientes com a Doença de Alzheimer e pacientes com lobectomia temporal têm pior desempenho em fluência verbal, devido a falhas na memória verbal (Rodrigues et al., 2008).

No teste de FV os erros devem ser cuidadosamente analisados, pois fornecem informações qualitativas de manifestações cognitivas, como repetições sem crítica, inclusão de palavras de itens de outras categorias, parafasias e outros. A FV reduzida também pode estar associada à perda da iniciativa ou à dificuldade em compreender e seguir regras (Butman, Allegri, Harris, \& Drake, 2000). A ordem de produção das palavras sugere o tipo de estratégia utilizada pelo sujeito. A eficiência no teste de FV requer a generalização de palavras dentro de subcategorias (agrupamentos) e trocas para uma nova subcategoria, quando a primeira se esgotar. Os agrupamentos dependem de processos de memória verbal e estão relacionados ao lobo temporal. Por outro lado, as trocas exigem flexibilidade mental e estão relacionadas ao lobo frontal e funções executivas (Hughes \& Bryan 2002).

Rodrigues et al. (2008) documentaram que a velocidade psicomotora é um dos fatores cognitivos importantes na FV, uma vez que a lentidão psicomotora da fala pode reduzir a quantidade de palavras. Sendo assim, o processo de resgate e o léxico semântico podem estar intactos, mas a velocidade psicomotora justificaria o pior desempenho.
A FV também é modulada por variáveis de natureza sócio-demográfica, como idade, escolaridade, sexo, e renda. O estudo de Carnero et al. (1999) objetivou averiguar o quanto a escolaridade influenciaria a fluência verbal em pacientes idosos sem demência. Os participantes do estudo apresentavam as seguintes características: tinham mais de 55 anos, não apresentavam queixas de memória, não tinham déficits cognitivos, e também não apresentavam suspeita de déficit funcional ou cognitivo, segundo os acompanhantes. Os pacientes foram classificados entre portadores e não portadores de patologias que poderiam gerar declínio cognitivo. Verificou-se que os sujeitos menos escolarizados apresentavam menor escore total no teste de fluência verbal e mencionavam menor variedade de animais. Sexo não correlacionou-se significativamente com FV. O diagnóstico mostrou uma associação significativa com FV no modelo de regressão ajustada. Concluiu-se que a escolaridade exerce influência significativa nas funções executivas.

No estudo brasileiro de Brucki, Malheiros, Okamoto e Bertolucci (1997) foram calculados pontos de corte que ajudariam a separar idosos cognitivamente preservados de idosos com risco elevado para as demências. Nove animais foi considerado um ponto de corte adequado para idosos com menos de 8 anos de escolaridade e 13 animais para idosos com mais de 8 anos de escolaridade. Caramelli, Carthery-Goulart, Porto, Charchat-Fichman e Nitrini (2007) também avaliaram a acurácia da FV animais para a detecção da doença de Alzheimer em diversas faixas de escolaridade. A FV apresentou ótima acurácia com os seguintes pontos de corte: nove animais para analfabetos, 12 animais entre um e sete anos de escolaridade, e 13 animais para 8 anos de escolaridade ou mais.

Em estudo mais recente Brucki e Rocha (2004) documentaram a influência da escolaridade, sexo e idade, nos escores do teste de fluência verbal. Foram avaliados: o número total de animais citados, o número de categorias de animais mencionadas, a quantidade de grupos de animais mencionados dentro de cada categoria, e quantidade de mudanças de categoria. Foram recrutados 257 participantes saudáveis, sendo 152 mulheres e 105 homens, com idade média de 49,4 anos e com nível educacional médio de 5,6 anos. Não houve influência significativa do sexo na pontuação do teste de fluência verbal, entretanto a idade e a escolaridade exerceram influência importante nos escores. Os participantes mais jovens e mais escolarizados geraram maior número de animais e maior número de categorais. A escolaridade foi a variável com maior influência no teste de FV categoria animais.

Em suma, os estudos sobre FV indicam que há forte influência de váriáveis sócio-demográficas no desempenho cognitivo. Baseando-se nesse contexto objetivouse no presente estudo investigar por meio de estudo epidemiológico realizado em um distrito da zona leste de São Paulo, o desempenho de idosos no teste de fluencia verbal e sua relação com idade, escolaridade, sexo e renda. 
Silva, T. B. L., Yassuda, M. S., Guimarães, V. V. \& Florindo, A. A. (2011). Fluência Verbal e Variáveis Sociodemográficas no Processo de Envelhecimento: Um Estudo Epidemiológico.

\section{Método}

\section{Participantes}

Participaram deste estudo de corte transversal de base domiciliar 383 idosos residentes em Ermelino Matarazzo, com idade igual ou superior a 60 anos. A amostragem foi probabilística, por conglomerados, em dois estágios. Foi realizado sorteio de 35 setores censitários do Distrito de Ermelino Matarazzo, na Zona Leste do Município de São Paulo, e a seguir realizou-se o recrutamento dos idosos residentes nos domicílios destes setores. As variáveis analisadas neste estudo fizeram parte do protocolo de pesquisa de um estudo maior (Salvador, Florindo, Reis, \& Costa, 2009). As características da amostra de idosos, cujos dados foram analisados neste estudo, estão descritas em Resultados.

Devido ao fato do último censo do Instituto Brasileiro de Geografia e Estatística (IBGE, 2000) ter sido realizado oito anos antes da pesquisa, após o sorteio dos 35 setores censitários, foi necessária a realização da contagem de todos os domicílios existentes em cada setor sorteado (arrolamento). O arrolamento foi realizado pelos entrevistadores participantes do estudo e se caracterizou pela contagem dos endereços tendo como referência os mapas e as indicações do IBGE (2000). Todos os endereços foram registrados, inclusive os imóveis comerciais, igrejas e templos, escolas, imóveis em construção e registro de todos os domicílios de cada setor, somente as residências ocupadas foram inclusas na coleta e foram procurados idosos em todos domicílios.

Para sortear os sujeitos no domicílio foi utilizada a metodologia de Kish (1965) que define tabelas aleatórias de acordo com o total de moradores. Cada domicílio recebeu uma tabela definindo qual sujeito deveria ser entrevistado, de acordo com o número de moradores idosos no domicílio. Foram estipuladas oito tabelas diferentes para que os sorteios mantivessem aleatoriedade. Dessa forma os moradores idosos dos domicílios foram ordenados de acordo com a idade e de maneira decrescente - do mais velho para o mais novo (Salvador, 2008).

Durante a coleta de dados, primeiramente o entrevistador identificava o domicílio sorteado (com endereço, número estipulado do domicílio e setor), listava o número total de moradores no domicílio (contando apenas os moradores com 60 anos ou mais) e identificava o sor-teado. Após o sorteio, o entrevistador marcava o dia e o horário para a entrevista que era realizada após o sujeito sorteado assinar o Termo de Consentimento Livre e Esclarecido.

Para participar da amostra do presente estudo os participantes deveriam apresentar idade igual ou superior a 60 anos, residir há pelo menos seis meses no município selecionado. Foram excluídos idosos que apresentaram doenças ou problemas que afetassem as atividades físicas de locomoção ou as atividades físicas de lazer até uma semana antes, da abordagem ou que apresentassem problemas de saúde mental, que impedissem o idoso de responder o questionário sozinho.

\section{Instrumentos}

Para o presente estudo foram selecionadas variáveis sócio-demográficas como sexo, idade, escolaridade e renda; e o teste de fluência verbal categoria animais.

Para o teste de fluência verbal, categoria animais foram calculados o total de animais gerados em um minuto, e sub-escores referentes aos animais produzidos a cada intervalo de 15 segundos. Os animais nomeados foram divididos em categorias (domésticos e fazenda, selvagens, insetos, peixes, pássaros, e répteis) segundo a metodologia proposta por Brucki e Rocha (2004). Foram calculados também: o número de categorias geradas, a quantidade de trocas de categorias, e uma medida de agrupamento. Nesta última medida era considerada a ocorrência de um grupo, quando o participante mencionava uma seqüência de três ou mais animais da mesma categoria, por exemplo, jacaré, lagarto e cobra.

\section{Aspectos Éticos}

O projeto de pesquisa foi aprovado pelo Comitê de Ética em Pesquisa da Faculdade de Saúde Pública da Universidade de São Paulo, parecer de número 1488. Antes de iniciar a avaliação cada participante da pesquisa assinou um Termo de Consentimento Livre e Esclarecido, e após a conclusão da entrevista recebeu orientações sobre atividade física e saúde e sobre os programas de atividade física desenvolvidos no bairro onde residem.

\section{Análises Estatísticas}

Todas as variáveis foram inicialmente submetidas a análises descritivas univariadas. Para descrever o perfil da amostra, segundo as diversas variáveis em estudo, foram feitas tabelas de freqüência das variáveis categóricas e estatísticas descritivas. Para comparação das variáveis nominais entre os grupos foram utilizados o teste QuiQuadrado, ou o teste exato de Fisher (na presença de valores esperados menores que cinco), ambos são testes estatísticos que avaliam a associação entre duas variáveis nominais.

Os dados cognitivos, variáveis do teste de fluência verbal, não apresentaram distribuição normal, por isso utilizou-se testes não paramétricos. Como os escores do teste de fluência verbal são variáveis contínuas, foi utilizado o teste de Kruskal-Wallis, quando foram comparados mais de dois grupos de amostras independentes, como, por exemplo, as faixas etárias. Para as comparações de duas amostras independentes, por exemplo, sexo, foi utilizado o Teste $U$ de Mann-Whitney (Bisquerra, Sarriera, \& Martinez, 2004). Para analisar a relação entre as variáveis numéricas foi utilizado o coeficiente de correlação de Spearman. O nível de significância adotado para os testes estatísticos foi de $5 \%$, ou seja, $p<0,05$.

Optou-se por realizar análise de regressão linear múltipla, visto que o desempenho cognitivo pode não ser explicado por um único agente causal, mas por uma multiplicidade de fatores. Em outras palavras, a variação de uma 
variável dependente deve ser testada em função de diversas variáveis explanatórias (Vieira, 2004). A análise de regressão múltipla foi realizada por meio de modelo linear generalizado (GLM) devido à amostragem complexa, a qual requer modelagem com estimações mais robustas e que incorporem os fatores de ponderação da amostra. A definição do modelo final foi feita baseada em pressupostos teóricos e nas variações do Bayesian Information Criterion (BIC). Foram testadas possíveis interações entre as variáveis e foi realizada análise de resíduos, para averiguar a adequação do modelo múltiplo final.

Os dados foram digitados duplamente no Programa Epidata versão 3.1 e para a validação dos dados foi utilizado o modo validate. Para análise estatística foi utilizado o programa computacional SPSS 15.0, módulo Complex Samples.

\section{Resultados}

Observa-se na Tabela 1 que a amostra continha maior presença de mulheres $(60,3 \%)$. Grande parte da amostra apresentava o ensino primário incompleto $(28,7 \%)$ ou o ensino primário completo $(25,6 \%)$. A pesquisa fez referência ao termo ensino primário, pois para os idosos, o primário refere-se aos primeiros quatro anos do que é chamado de ensino fundamental atualmente. Quanto à renda líquida familiar per capita, em média os idosos recebiam $\mathrm{R} \$ 509,00(D P=618,80)$. Destaca-se que este dado não foi obtido para 81 participantes que ou recusaram-se a responder a esta questão, ou não tinham certeza quanto à renda familiar.

Tabela 1

Perfil Sócio-demográfico dos Idosos Participantes, Distrito de Ermelino Matarazzo, Zona Leste do Município de São Paulo, 2008

\begin{tabular}{lcc}
\hline Variável & $n(383)$ & $\%$ \\
\hline Sexo & & \\
$\quad$ Feminino & 231 & 60,3 \\
$\quad$ Masculino & 152 & 39,7 \\
Idade & & \\
$\quad$ Média $(D P)$ & $692(7,3)$ & - \\
$\quad$ Mediana (Mín-Max) & $68,0(60,0-97,0)$ & - \\
Escolaridade & & \\
$\quad 0-4$ anos & 280 & 73,1 \\
$5-8$ anos & 50 & 13,1 \\
9 ou mais & 53 & 13,8 \\
Trabalha & 84 & 21,9 \\
$\quad$ Sim & 299 & 78,1 \\
$\quad$ Não & & \\
Renda Familiar per Capita & \\
$\quad$ Média (DP) & $509,0(618,8)$ & - \\
Mediana (Mín-Máx) & $350,0(54,3-7.500,0)$ & - \\
\hline
\end{tabular}

Nota. Números absolutos na amostra não-ponderada.

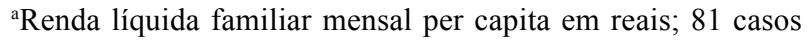
ignorados.
Os idosos da amostra falaram em média 11,1 animais $(D P=3,8)$. Os animais domésticos, de fazenda e selvagens foram os mais citados, e na média os idosos citaram três categorias de animais $(D P=1,0)$. Houve maior produção de animais nos dois primeiros intervalos de 15 segundos, como observa-se na Tabela 2 .

Tabela 2

Médias e Desvios-padrão (DP) para as Variáveis do Teste de Fluência Verbal para a Amostra Total $(n=383)$, Distrito de Ermelino Matarazzo, Zona Leste do Municipio de São Paulo, 2008

\begin{tabular}{lccc}
\hline & $M$ & $D P$ & $\begin{array}{c}\text { Min- } \\
\text { Max }\end{array}$ \\
\hline Intervalo 1 & 5,05 & 1,82 & $0-11$ \\
Intervalo 2 & 2,53 & 1,48 & $0-8$ \\
Intervalo 3 & 1,81 & 1,34 & $0-7$ \\
Intervalo 4 & 1,58 & 1,44 & $0-7$ \\
Total de animais & 11,11 & 3,79 & $0-23$ \\
Número de categorias & 2,95 & 1,03 & $0-6$ \\
Número de grupos & 1,52 & 0,89 & $0-5$ \\
Mudanças de categoria & 3,81 & 2,36 & $0-13$ \\
\hline
\end{tabular}

Foram testadas as diferenças na quantidade de animais gerados entre sexo, faixas etárias e escolaridade. Os homens mencionaram maior número de animais nos intervalos 1 e 3, emitiram maior quantidade de animais no total geral, e formaram maior quantidade de grupos e animais, quando comparados às mulheres (ver Tabela 3 ).

Tabela 3

Médias e Desvios-padrão (DP) para as Variáveis do Teste de Fluência Verbal segundo Sexo, Distrito de Ermelino Matarazzo, Zona Leste do Município de São Paulo, 2008

\begin{tabular}{lccccc}
\hline & \multicolumn{2}{c}{$\begin{array}{c}\text { Homens } \\
(n=152)\end{array}$} & \multicolumn{4}{c}{$\begin{array}{l}\text { Mulheres } \\
(n=231)\end{array}$} \\
\hline Variável & $M$ & $D P$ & $M$ & $D P$ & $p^{*}$ \\
\hline Intervalo 1 & 5,40 & 1,89 & 4,82 & 1,74 & 0,001 \\
Intervalo 2 & 2,51 & 1,41 & 2,54 & 1,53 & 0,985 \\
Intervalo 3 & 2,01 & 1,45 & 1,68 & 1,25 & 0,031 \\
Intervalo 4 & 1,73 & 1,52 & 1,48 & 1,37 & 0,127 \\
Total de animais & 11,74 & 4,03 & 10,70 & 3,57 & 0,007 \\
$\begin{array}{l}\text { Número de } \\
\text { categorias }\end{array}$ & 2,93 & 1,03 & 2,95 & 1,03 & 0,902 \\
Número de grupos & 1,64 & 0,97 & 1,45 & 0,84 & 0,040 \\
$\begin{array}{l}\text { Mudanças } \\
\text { de categoria }\end{array}$ & 3,96 & 2,43 & 3,72 & 2,32 & 0,385 \\
\hline
\end{tabular}

*Teste $U$ Mann-Whitney. 
Silva, T. B. L., Yassuda, M. S., Guimarães, V. V. \& Florindo, A. A. (2011). Fluência Verbal e Variáveis Sociodemográficas no Processo de Envelhecimento: Um Estudo Epidemiológico.

Na Tabela 4 são apresentadas as comparações dos escores da fluência verbal para cada faixa etária separada- mente. No intervalo 1, total de animais e mudança de categoria, os indivíduos pertencentes à faixa etária mais jovem obtiveram desempenho superior.

Tabela 4

Médias e Desvios-padrão para as Variáveis do Teste de Fluência Verbal segundo Faixa Etária, Distrito de Ermelino Matarazzo, Zona Leste do Município de São Paulo

\begin{tabular}{lccccc}
\hline & \multicolumn{2}{c}{$\begin{array}{c}\text { 60-69 anos } \\
(n=217)\end{array}$} & \multicolumn{2}{c}{$\begin{array}{c} \\
(n=166)\end{array}$} & \\
\hline Variável & $M$ & $D P \pm$ & $M$ & $D P \pm$ & $p^{*}$ \\
\hline Intervalo 1 & 5,29 & 1,90 & 4,73 & 1,67 & 0,003 \\
Intervalo 2 & 2,59 & 1,48 & 2,45 & 1,49 & 0,312 \\
Intervalo 3 & 1,90 & 1,37 & 1,69 & 1,29 & 0,174 \\
Intervalo 4 & 1,60 & 1,47 & 1,55 & 1,40 & 0,869 \\
Total de animais & 11,58 & 3,82 & 10,50 & 3,67 & 0,005 \\
Número de categorias & 3,00 & 1,05 & 2,87 & 1,00 & 0,182 \\
Número de grupos & 1,58 & 0,93 & 1,45 & 0,85 & 0,186 \\
Mudanças de categoria & 3,99 & 2,30 & 3,58 & 2,43 & 0,018
\end{tabular}

*Teste $U$ Mann-Whitney.

Em relação à escolaridade, os sujeitos pertencentes ao grupo mais escolarizado (cinco anos ou mais) obtiveram melhor desempenho nas seguintes variáveis: intervalo 1, total de categorias, mudança de categoria e total de animais (Tabela 5). Em relação à variável renda familiar per capita, não foi encontrada diferença significativa no desempenho do teste de fluência verbal, quando estratificouse a amostra em três grupos, entre $\mathrm{R} \$ 150,00$ e $\mathrm{R} \$ 560,00$ entre $R \$ 561,00$ e $R \$ 1.000,00$, e R $\$ 1.001,00$ e mais (dados não apresentados em tabelas).

Tabela 5

Médias e Desvios-padrão para as Variáveis do Teste de Fluência Verbal segundo Escolaridade, Distrito de Erme-lino Matarazzo, Zona Leste do Município de São Paulo

\begin{tabular}{|c|c|c|c|c|c|}
\hline \multirow[b]{2}{*}{ Variável } & \multicolumn{2}{|c|}{$\begin{array}{l}0-4 \text { anos } \\
(n=280)\end{array}$} & \multicolumn{2}{|c|}{$\begin{array}{c}5 \text { anos ou mais } \\
(n=103)\end{array}$} & \multirow[b]{2}{*}{$p^{*}$} \\
\hline & $M$ & $D P \pm$ & $M$ & $D P \pm$ & \\
\hline Intervalo 1 & 4,93 & 1,83 & 5,38 & 1,77 & 0,027 \\
\hline Intervalo 2 & 2,46 & 1,49 & 2,73 & 1,43 & 0,146 \\
\hline Intervalo 3 & 1,77 & 1,28 & 1,91 & 1,50 & 0,687 \\
\hline Intervalo 4 & 1,49 & 1,38 & 1,81 & 1,56 & 0,097 \\
\hline Total de animais & 10,73 & 3,69 & 12,14 & 3,88 & 0,006 \\
\hline Número de categorias & 2,83 & 0,97 & 3,26 & 1,11 & 0,001 \\
\hline Número de grupos & 1,51 & 0,88 & 1,57 & 0,95 & 0,666 \\
\hline Mudanças de categoria & 3,56 & 2,26 & 4,51 & 2,52 & 0,001 \\
\hline
\end{tabular}

*Teste U Mann-Whitney.

Por meio da Tabela 6, observa-se que na amostra $(n=383)$, a idade se correlacionou à variável de fluência verbal. Observou-se que quanto maior a idade menor a produção de animais nos primeiros 15 segundos. Nova- mente, a magnitude das correlações foi pequena. Em relação à escolaridade, houve correlação significativa com as variáveis: intervalo 1 , total de categorias, total de animais e total de mudanças de categorias. 
Tabela 6

Correlações entre Escores de Fluência Verbal e Variáveis Sócio-demográficas para a Amostra Total ( $n=383)$, Distrito de Ermelino Matarazzo, Zona Leste do Município de São Paulo, 2008

\begin{tabular}{lrrrrrr}
\hline & \multicolumn{2}{c}{ Idade } & \multicolumn{2}{c}{ Escolaridade } & \multicolumn{2}{c}{ Renda $^{\text {a }}$} \\
\hline Variáveis da Fluência Verbal & $r^{b}$ & $p$ & $r^{b}$ & $p$ & $r^{b}$ & $p$ \\
\hline Intervalo 1 & $-0,17$ & 0,001 & 0,11 & 0,039 & 0,11 & 0,052 \\
Intervalo 2 & $-0,03$ & 0,506 & 0,08 & 0,135 & 0,07 & 0,249 \\
Intervalo 3 & $-0,05$ & 0,319 & 0,02 & 0,655 & 0,03 & 0,656 \\
Intervalo 4 & $-0,01$ & 0,911 & 0,03 & 0,528 & 0,06 & 0,328 \\
Total de animais & $-0,14$ & 0,006 & 0,12 & 0,024 & 0,10 & 0,072 \\
Número de Categorias & $-0,08$ & 0,137 & 0,13 & 0,009 & 0,09 & 0,129 \\
Número de Grupos & $-0,10$ & 0,044 & 0,05 & 0,353 & 0,00 & 0,932 \\
Mudança de categorias & $-0,08$ & 0,098 & 0,14 & 0,006 & 0,08 & 0,180 \\
\hline
\end{tabular}

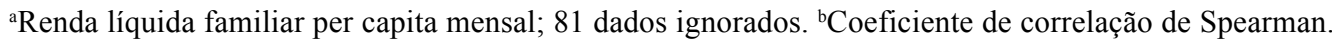

A análise de regressão linear (GLM) foi utilizada para estimar o efeito de cada uma das variáveis sociodemográficas sobre o escore de fluência verbal. O modelo ajustado é estatisticamente significativo (BIC $=2131,54$; $\left.X^{2}=28,45, \mathrm{gl}=3, p=0,000\right)$, e as estimativas dos coefi- cientes do modelo são apresentadas na Tabela 7. Observou-se que sexo e escolaridade foram as variáveis que mantiveram significância estatística com o escore de fluência verbal, mesmo após o ajuste no modelo multivariado. Não houve interações entre as variáveis presentes no modelo final ajustado.

Tabela 7

Análise de Regressão Linear Múltipla do Escore de Fluência Verbal segundo Variáveis Sócio-demográficas em População Idosa, Distrito de Ermelino Matarazzo, Zona Leste do Municipio de São Paulo, 2008

\begin{tabular}{lrrrr}
\hline & Modelo Bruto & \multicolumn{2}{c}{ Modelo Ajustado $^{\mathrm{b}}$} \\
\hline$\beta^{\mathrm{a}}$ & $p$ & $\beta^{\mathrm{a}}$ & $p$ \\
\hline Sexo $^{\mathrm{c}}$ & 1,006 & 0,023 & 0,946 & 0,028 \\
Idade & $-0,066$ & 0,022 & $-0,052$ & 0,075 \\
Escolaridade & 0,253 & 0,000 & 0,233 & 0,000 \\
\hline
\end{tabular}

Nota. Modelo linear generalizado multivariado.

${ }^{\text {a}}$ Valores padronizados de $\beta .{ }^{b}$ Ajustado para variáveis apresentadas na tabela. ${ }^{c}$ Feminino(categoria de referência) versus masculino.

\section{Discussão}

O presente estudo objetivou averiguar se variáveis sócio-demográficas estariam associadas ao desempenho no teste de fluência verbal categoria animais. Adicionalmente, investigou-se se as variáveis do teste de fluência verbal (desempenho nos quatro intervalos de tempo, número de categorias, mudanças de categorias e número de grupos mencionados) poderiam estar associadas às variáveis sócio-demográficas. As análises univariadas e multivariadas indicaram que o desempenho nesta tarefa é modulado pelas variáveis idade, escolaridade e sexo.

Os resultados do estudo indicam que os participantes mencionaram em média onze animais, resultado semelhante aos encontrados em outros estudos realizados em âmbito ambulatorial e na comunidade, que também ava- liaram idosos sem demência e de diferentes faixas de escolaridade (Caramelli et al., 2007; Radanovic et al., 2009). A média de número de grupos e categorias mencionadas foi semelhante aos resultados apresentados no estudo de Brucki e Rocha (2004).

Os idosos mais escolarizados apresentaram melhor desempenho em fluência verbal total e no primeiro intervalo de 15 segundos, no número de categorias citadas, e no número de mudanças de categorias. Estes resultados são semelhantes aos encontrados em estudos anteriores (Bolla, Gray, Resnick, Galante, \& Kawas, 1998; Brucki \& Rocha, 2004; Carnero et al., 1999; Kempler, Teng, Dick, Taussig, \& Davis, 1998). As correlações também apontaram forte associação entre escolaridade e as variáveis do teste de fluência verbal. Estes dados sugerem que os idosos com maior escolaridade são mais rápidos no aces- 
so semântico, conseguem pensar em um maior número de categorias e alternar entre elas, como estratégia para resgatar maior número de animais.

Os resultados indicaram que a idade também esteve associada ao desempenho no teste de fluência verbal. Quanto maior a idade menor a pontuação no total de animais, no primeiro intervalo, e no número de mudanças de categorias. Esses achados estão em concordância com os resultados de Acevedo et al. (2000) e Kempler et al. (1998) que também detectaram influência da idade em seus estudos. Esses resultados foram contrários aos encontrados por Benito-Cuadrado, Esteba-Castilho, Bohm, Cejudo-Bolivar e Peña-Casanova (2002) que não verificaram em sua amostra $(n=445)$ de diferentes faixas etárias (jovens, adultos e idosos) correlação estatística significativa entre idade e o desempenho no teste de fluência verbal categoria animais. Porém, ao correlacionarem escolaridade e fluência verbal detectaram significância estatística, em consonância com a literatura cognitiva gerontológica.

Os homens mencionaram maior quantidade de animais quando comparados às mulheres, no desempenho total, nos intervalos 1 e 3, e no número de grupos produzidos. Estes dados concordam com achados de Kempler et al. (1998), quando verificou-se melhor desempenho entre os homens, entretanto, discordam dos resultados apresentados pela maioria do estudos sobre fluência verbal. Lezak et al. (2004) documentam que as mulheres têm melhor desempenho em fluência com restrição fonológica (categoria animais e frutas). Estes autores pontuam que as mulheres mantêm desempenho superior no processo de envelhecimento normal. Crossley, D'Arcy e Rawson (1997) relataram que as mulheres apresentaram melhor desempenho em fluência com restrição semântica, porém, os homens superaram as mulheres em fluência com restrição fonológica. No presente estudo, as diferenças a favor dos homens talvez estejam associadas à escolaridade mais alta dos mesmos, entretanto, sexo destacou-se como preditor significativo de fluência verbal no modelo de regressão que incluiu escolaridade.

No presente trabalho, observou-se que as categorias de animais mais mencionados foram os domésticos e de fazenda e os selvagens, como em Brucki e Rocha (2004) e Carnero et al. (1999). Esses estudos documentaram forte influência do ambiente nos tipos de animais gerados na tarefa de fluência verbal entre indivíduos idosos, principalmente em idosos residentes em área rurais.

Em suma, os resultados apresentados sugerem que o desempenho em fluência verbal sofre influência significativa de variáveis sócio-demográficas, a saber, idade, escolaridade e sexo. Estes dados sugerem que a interpretação clínica do resultado nesta prova, que com freqüência é utilizada para rastrear quadros demenciais, deve considerar as características sócio-demográficas do paciente. Estudos da acurácia desta tarefa para a detecção de doenças com repercussão no funcionamento cognitivo devem estratificar a amostra em faixas de escolaridade, idade e por sexo. Uma limitação do presente estudo refere-se à variável renda, pois em inquéritos de base populacional, é usual a perda de 20 a $30 \%$ dos dados desta variável, pois algumas pessoas preferem não revelar este dado.

É importante ressaltar ainda que esse estudo, diferentemente dos estudos nacionais que o antecederam, não está sujeito ao viés característico de populações clínicas e ambulatoriais, devido à utilização de metodologia epidemiológica, sendo assim mais representativo da população brasileira.

\section{Referências}

Acevedo, A., Loewenstein, D. A., Barker, W. W., Harwood, D. G., Luis, C., Bravo, M., et al. (2000). Category fluency test: Normative data for English- and Spanish-speaking elderly. Journal of the International Neuropsychological Society, 6 , 760-769.

Benito-Cuadrado, M. M., Esteba-Castilho, S., Bohm, P., CejudoBolivar, J., \& Pena-Casanova, J. (2002). Verbal fuency of animals: A normative and predictive study in a Spanish population, Journal of Clinical and Experimental Neuropsychology, 24(8), 1117-1122.

Bolla, K. I., Gray, S., Resnick, S., Galante, R., \& Kawas, C. (1998). Category and letter fluency in highly educated older adults. The Clinical Neuropsychologist, 12(3), 330-338.

Bisquerra, R., Sarriera, J. C., \& Martinez, F. (2004). Introdução à Estatística: Enfoque informático com o pacote estatístico SPSS. Porto Alegre, RS: Artmed.

Brucki, S. M. D., Malheiros, S. M. F., Okamoto, I. H., \& Bertolucci, P. H. F. (1997). Dados normativos para o teste de fluência verbal categoria animais em nosso meio. Arquivos de Neuropsiquiatria, 55(1), 56-61.

Brucki, S. M. D., Nitrini, R., Caramelli, P., Bertolucci, P. H. F., \& Okamoto, I. H. (2003). Sugestões para o uso do MiniExame do Estado Mental. Arquivos de Neuro-psiquiatria, 61(3-B), 777-781.

Brucki, S. M. D., \& Rocha, M. S. G. (2004). Category fluency test: Effects of age, gender and education on total scores, clustering, in Brazilian Portuguese-speacking subjeticts. Brazilian Journal of Medical and Biological Research, 37(12), 1771-1777.

Butman, J., Allegri, R. F., Harris, P., \& Drake, M. (2000). Fluência verbal en español: datos normativos en Argentina. Medicina, 60(5/1), 561-4.

Caramelli, P., Carthery-Goulart, M. T., Porto, C. S., CharchatFichman, H., \& Nitrini, R. (2007). Category fluency as a screening test for Alzheimer Disease in illiterate and and literate patients. Alzheimer Disease \& Associated Disorders, 21(1), 65-67.

Carnero, C., Lendínez, A., Maestre J., \& Zunzunegui, M. V. (1999). Fluencia verbal semántica en pacientes neurológicos sin demencia y bajo nivel educativo. Revista de Neurología, 28(9), 858-862.

Crossley, M., D’Arcy, C., \& Rawson, N. S. (1997). Letter and category fluency in community-dwelling Canadian seniors: A comparison of normal participants to those with dementia of the Alzheimer or vascular type. Journal of Clinical and Experimental Neuropsychology, 19, 52-62. 
Gaillard, W. D., Hertz-Pannier, L., Mott, S. H., Barnett, A. S., LeBihan, D., \& Theodore, W. H. (2003). Developmental aspects of language processing: fMRI of verbal fluency in children and adults. Human Brain Mapping, 18, 176-185.

Hughes, D. L., \& Bryan J. (2002). Adult age differences in strategy use during verbal fluency performance. Journal of Clinical and Experimental Neuropsychology, 24(5), 642-54.

Instituto Brasileiro de Geografia e Estatística. (2000). Estimativas de população. Retrieved February 20, 2009, from http:/ /www.ibge.gov.br/home/estatistica/populacao/

Kempler, D., Teng, E. L., Dick, M., Taussig, I. M., \& Davis, D. S. (1998). The effects of age, education, and ethnicity on verbal fluency. Journal of the International Neuropsychological Society, 4(6), 531-538.

Kristensen, C. H. (2006). Funções executivas e envelhecimento. In M. A. A. P. Parente (Ed.), Cognição e envelhecimento (pp. 96-111). Porto Alegre, RS: Artmed.

Kish, L. (1965). Survey sampling. New York: Wiley.

Lezak, M. D., Howieson, D. B., \& Loring, D. W. (2004). Neuropsychological assessment $\left(4^{\text {th }} \mathrm{ed}\right.$.). New York: Oxford University Press.

Magila, C., \& Caramelli, P. (2001). Funções executivas no idoso. In O. V. Forlenza \& P. Caramelli (Eds.), Neurosiquiatria Geriátrica (pp. 517-525). São Paulo, SP: Atheneu.

Neri, A. L. (2006). Envelhecimento cognitivo. In E. V. Freitas, L. Py, F. A. X. Cançado, J. Doll, M. L. Gorzoni, \& S. M. Rocha (Eds.), Tratado de Geriatria e Gerontologia (pp. 12361244). Rio de Janeiro, RJ: Guanabra Koogan.

Nitrini, R., Caramelli, P., Bottino, C. M. C., Damasceno, B. P., Brucki, S. M. D., \& Anghinah, R. (2005). Diagnóstico de doença de Alzheimer no Brasil: Avaliação cognitiva e funcional. Recomendações do Departamento Científico de Neurologia Cognitiva e do Envelhecimento da Academia Brasileira de Neurologia. Arquivos de Neuro-Psiquiatria, 63(3A), 720-727.

Radanovic, M., Diniz, B. S., Mirandez, R. M., Novaretti, T. M. S., Flaks, M. K., Yassuda, M. S., et al. (2009). Verbal fluency in the detection of mild cognitive impairment and Alzheimer's disease among Brazilian Portuguese speakers: The influence of education, International Psychogeriatrics, 21, 1-7.

Rodrigues, A. B., Yamashita, E. T., \& Chiappetta, A. L. M. L. (2008). Teste de fluência verbal no adulto e no idoso: Verificação da aprendizagem verbal. Revista CEFAC, 10(4), 443451.

Salvador, E. P. (2008). Atividade física e sua associação com o ambiente em idosos residentes do distrito de Ermelino Matarazzo, zona leste do município de São Paulo. Dissertação de Mestrado não-publicada, Faculdade de Saúde Pública, Universidade de São Paulo, SP.

Salvador, E. P., Florindo, A. A., Reis, R. S., \& Costa, E. F. (2009). Percepção do ambiente e prática de atividade física no lazer entre idosos. Revista de Saúde Pública, 43(6), 972980.

Vieira, S. (2004). Tópicos avançados em Bioestatística: Testes não-paramétricos, tabelas de contingência e análises de regressão (2. ed. rev.). Rio de Janeiro, RJ: Campus.

West, R. (1996). An application of prefrontal cortex function theory to cognitive aging. Psychological Bulletin, 120(2), 272-292.

Yassuda, M. S., \& Abreu, V. P. S. (2011). Avaliação cognitiva em gerontologia. In E. V. Freitas, L. Py, F. A. X. Cançado, J. Doll, M. L.Gorzoni, \& S. M. Rocha (Eds.), Tratado de Geriatria e Gerontologia (pp. 1486-1493). Rio de Janeiro, RJ: Guanabra Koogan. 